\title{
AN ELEMENTARY SOLUTION OF THE MONOTONE MAPPING PROBLEM
}

\author{
JOSEPH MARTIN AND IRA ROSENHOLTZ
}

ABSTRACt. A simple example of a monotone, noncompact mapping from $\mathbf{R}^{\mathbf{3}}$ to $\mathbf{R}^{3}$ is constructed.

We let $\mathbf{R}^{n}$ denote Euclidean $n$-dimensional space with the usual topology, and we use the word map as an abbreviation for continuous function.

A map is monotone if and only if point inverses are compact and connected, and is compact if and only if inverse images of compact sets are compact.

In 1959, Whyburn [5] proved that any monotone map from $\mathbf{R}^{2}$ onto itself is necessarily compact, and asked if the same result held for $\mathbf{R}^{n}$. In 1969, a negative answer for $n \geqslant 4$ was given by Glaser [4], and later that year, R. H. Bing [1] produced a monotone, noncompact map from $\mathbf{R}^{3}$ onto itself, completely settling the question. Bing's argument is ingenious and difficult. The idea is roughly as follows: One-to-one maps from $\mathbf{R}_{+}^{3}$ (the closed upper half of $\mathbf{R}^{3}$ ) onto $\mathbf{R}^{3}$ were known ([2], [3]). Such maps are necessarily noncompact. Then if one could find a monotone map from $\mathbf{R}^{3}$ onto $\mathbf{R}_{+}^{3}$, its composition with a one-to-one map from $\mathbf{R}_{+}^{3}$ onto $\mathbf{R}^{3}$ would produce a monotone, noncompact map from $\mathbf{R}^{3}$ onto itself. In order to construct a monotone map from $\mathbf{R}^{3}$ onto $\mathbf{R}_{+}^{3}$, Bing proved the following lemma, which is the crux of the matter.

LEMMA. There is a map $f$ from the 3-cell $D$, onto itself, with the following properties:

(1) $f$ is monotone,

(2) if $y \in D$, then $f^{-1}(y)$ intersects the boundary of $D$, and

(3) there is a point $q$ on the boundary of $D$ whose inverse image is a single point.

On the basis of the above lemma it is a relatively easy matter to produce the desired map. Consider the 3-sphere $S^{3}$ as the union of two copies of $I^{3}$ attached along their boundaries by the identity map. Apply the map $f$ of the lemma to each copy of $I^{3}$. This yields a monotone map from $S^{3}$ onto $I^{3}$ which induces a monotone map from $S^{3}-\left\{f^{-1}(q)\right\}\left(\cong \mathbf{R}^{3}\right)$ onto $I^{3}-\{q\}$ $\left(\cong \mathbf{R}_{+}^{3}\right)$.

The purpose of this paper is to provide an elementary proof of the

Received February 3, 1978.

AMS (MOS) subject classifications (1970). Primary 54C10, 57A10.

Key words and phrases. Monotone Mapping Problem, monotone map, compact map.

(c) American Mathematical Society 1978 
preceding lemma. We would like to thank Professors R. H. Bing and C. E. Burgess for a careful reading of the first draft of this paper. They pointed out an error and suggested several improvements which we have incorporated into this final manuscript.

Proof of Lemma. Step 0. We begin with a trivial modification of the usual Cantor function. Let $C$ denote the usual middle thirds Cantor set in $[0,1]$. The function $g: C \rightarrow[0,1]$ defined by

$$
g\left(\sum_{n=1}^{\infty} \frac{a_{n}}{3^{n}}\right)=\sum_{n=1}^{\infty} \frac{a_{n}}{2^{n+1}}
$$

has a unique continuous extension to a map $g_{0}:[-1,2] \rightarrow[0,1]$ which is constant on each subinterval of $[-1,2]-C . g_{0}$ has the following properties:

(a) $g_{0}$ is monotone,

(b) if $y \in[0,1]$, then $g_{0}^{-1}(y)$ intersects $C$.

Step 1. Let $g_{1}:[-1,2]^{3} \rightarrow[0,1]^{3}$ be defined by $g_{1}(x, y, z)=\left(g_{0}(x), g_{0}(y)\right.$, $\left.g_{0}(z)\right) . g_{1}$ has the following properties:

(a) $g_{1}$ is monotone,

(b) if $y \in[0,1]^{3}$, then $g_{1}^{-1}(y)$ intersects $C \times C \times C$.

Step 2. Let $K$ denote the Cantor set $C \times C \times C \subset \operatorname{Int}\left([-1,2]^{3}\right)$. $K$ is a tame Cantor set. Let $g_{2}$ be a homeomorphism from $[-1,2]^{3}$ onto itself such that $g_{2}^{-1}(K)=\{0\} \times C \times\{0\}$, and which is the identity on the boundary.

Step 3 . In this step we construct a map $g_{3}$ from $[0,1]^{3}$ onto $[-1,2]^{3}$ such that

(a) $g_{3}$ is monotone,

(b) $g_{3}^{-1}(\{0\} \times C \times\{0\})$ is a subset of the boundary of $[0,1]^{3}$, and

(c) $g_{3}$ takes the left and right faces of $[0,1]^{3}$ homeomorphically onto the left and right faces respectively of $[-1,2]^{3}$ so that $g_{3}(0, y, z)$ and $g_{3}(1, y, z)$ have the same last two coordinates.

Consider the following diagram.

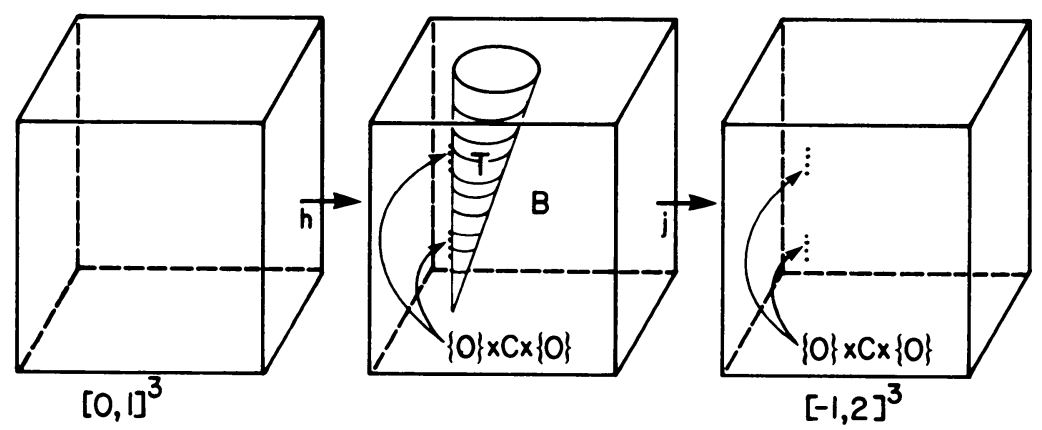

Let $B$ denote the 3-cell which is the complement of the cone shaped tunnel $T$ in $[-1,2]^{3}$. Let $h$ be a homeomorphism from $[0,1]^{3}$ onto $B$ which satisfies (c). Notice that horizontal planes intersect the boundary of $T$ in simple closed 
curves. Let $j$ be the map from $B$ onto $[-1,2]^{3}$ which shrinks each of these simple closed curves to points. Then the map $g_{3}$ is $(j \circ h)$.

Step 4. Let $g$ be the map from $[0,1]^{3}$ onto $[0,1]^{3}$ defined by $g=$ $\left(g_{1} \circ g_{2} \circ g_{3}\right)$. Then

(a) $g$ is monotone and

(b) if $y \in[0,1]^{3}$ then $g^{-1}(y)$ intersects the boundary of $[0,1]^{3}$.

At this point we have a map satisfying conditions (1) and (2) of the lemma. To satisfy (3) we glue countably many copies of $g$ together as in the following diagram.

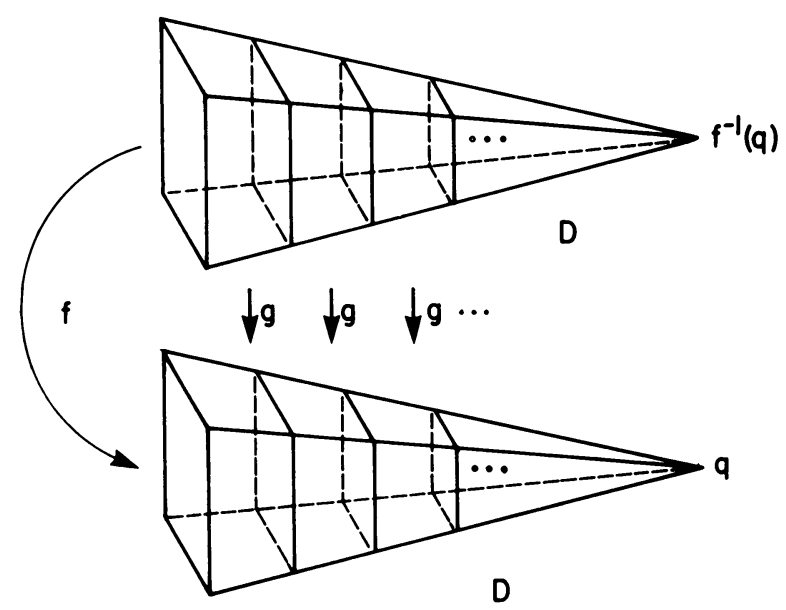

This completes the proof.

\section{REFERENCES}

1. R. H. Bing, The monotone mapping problem, Topology of Manifolds (J. C. Cantrell and C. H. Edwards, eds.), Markham, Chicago, pp. 99-115.

2. R. H. Bing and Joseph Martin, One-to-one maps of $E_{+}^{3}$, Proc. Binghamton Topology Conf., 1972.

3. L. C. Glaser, Dimension lowering monotone non-compact mappings of $E^{n}$, Fund. Math. 58 (1966), 177-181.

4. Monotone non-compact mappings of $E^{r}$ onto $E^{k}$ for $r \geqslant 4$ and $k>3$, Proc. Amer. Math. Soc. 23 (1969), 282-286.

5. G. T. Whyburn, Compactness of certain mappings, Amer. J. Math. 81 (1959), 306-314.

Department of Mathematics, University of Wyoming, Laramie, Wyoming 82071 